\section{Biomarkers to exclude the diagnosis of ventilator-associated pneumonia}

\author{
Michael A Matthay
}

Ventilator-acquired pneumonia (VAP) is an important clinical problem that has been associated with substantial morbidity and mortality. ${ }^{1}$ However, the diagnosis of VAP can be particularly difficult, ${ }^{2-4}$ and surveillance programmes have not been markedly successful. ${ }^{56}$ The diagnosis of VAP can be difficult, in part, because new radiographic infiltrates can be secondary to aspiration, acute respiratory distress syndrome (ARDS) without pneumonia, or atelectasis. ${ }^{7}$ Typical criteria for the diagnosis of VAP include a new radiographic infiltrate, the presence of purulent tracheal secretions, hypothermia or hyperthermia, and/ or a low or elevated peripheral white blood cell count. Some investigators and clinicians have used quantitative cultures of bronchoalveolar lavage (BAL) or tracheal aspirates with different thresholds of $>10^{3}$ or $>10^{4}$ colony-forming units to confirm the diagnosis with microbiological criteria. Pseudomonas aeruginosa and Staphylococcus aureus are the most common causes of VAP. Clinicians are usually compelled to initiate empiric antibiotic therapy in most patients with suspected VAP, pending a clinical response or the results of the microbiology cultures. However, quantitative culture results usually require $24-48 \mathrm{~h}$. Thus, it would be helpful to have a method for either more rapidly diagnosing VAP, or at least excluding VAP that could be accomplished within a few hours after suspecting the diagnosis.

In this issue of Thorax, Hellyer et $a l^{8}$ have reported the results of an interesting and well done clinical study to test for the potential value of measuring biomarkers in BAL that may be useful in excluding pneumonia in patients with suspected VAP. The study was multicentre and prospective and was carried out in 12 intensive care units. VAP was diagnosed by bronchoscopy with culture of a pathogen in the bronchoalveolar lavage fluid at $>10^{4}$ colony-forming units. Several biomarkers were measured, including interleukin-1 $\beta \quad$ (IL-1 $\beta), \quad$ interleukin-8

Correspondence to Professor Michael A Matthay, Departments of Medicine and Anesthesia,

Cardiovascular Research Institute, University of California, 505 Parnassus Ave, M-917, Box 0624, San Francisco, CA 94143-0624, USA; michael. matthay@ucsf.edu
(IL-8), matrix metalloproteinase (MMP)-8, MMP-9 and human neutrophil elastase. There were 150 patients included in the ultimate analysis, 53 patients (35\%) with VAP and 97 patients (65\%) in the non-VAP group. In terms of baseline data, the VAP group was significantly associated with less frequent use of antibiotics prior to the BAL. Gram-negative bacteria accounted for $60 \%$ of the organisms and Gram-positive accounted for $30 \%$ and fungi $10 \%$. All five of the biomarkers were significantly higher in the patients with confirmed VAP. The investigators constructed receiver-operating curves analyses for all five biomarkers to determine the diagnostic utility and optimum cut-off points. The results of further analyses indicated that diagnosis was optimal for a combination of IL- $1 \beta$ and IL- 8 with an area under the curve of 0.81 . A high negative predictive value was achieved with either IL-1 $\beta$ or IL- 8 for excluding VAP. The authors then further tested the findings by applying the biomarker cut-offs to a previously published derivation cohort and found that the sensitivity for the IL-1 $\beta$ and IL-8 combination was $94 \%$, specificity $56 \%$ and negative predictive value $97 \%$, with an area under the curve of 0.85 . Thus, the results provided impressive results that the measurement of IL- $1 \beta$ and IL- 8 could exclude VAP in a large fraction of the patients that were studied.

These results are interesting and provocative, suggesting that it might be possible to enhance current clinical diagnostic efforts to exclude VAP at an earlier phase in the patient's clinical course, thus preventing the unnecessary addition of new antibiotics to treat suspected VAP. The authors should be commended for doing a well-planned scientific study that included multiple sites. From a practical standpoint, in order to further test the utility of this approach, it would be necessary to have point-of-care assays for IL- $1 \beta$ and IL- 8 that could be processed rapidly in the clinical setting within $2-4 \mathrm{~h}$.

There is some earlier work on the potential use of protein biomarkers in BAL in patients with VAP from $P$ aeruginosa. In one study, plasminogen-activator inhibitor 1 (PAI-1) concentrations were correlated with higher mortality in ventilated patients with positive cultures for Paeruginosa, and also correlated with the secretion of type III exotoxins by $P$ aeruginosa. ${ }^{9}$ In that study, PAI-1 concentrations in patients with ARDS were significantly higher than the concentrations measured in patients without ARDS. However, a significant difference between survivors and nonsurvivors in elevated BAL fluid PAI-1 concentrations persisted even after exclusion of patients with ARDS. However, in that study, PAI-1 was not useful for distinguishing patients with and without VAP. Several other biomarkers have been tested for the diagnosis of VAP without much success. ${ }^{10}$

The most important limitation in the current study by Hellyer et $a l^{8}$ relates to the need to do fiberoptic bronchoscopy and lavage. The use of lavage was rational in this study because the investigators were able to direct the bronchoscope to the areas of the lung that showed maximal or substantial pulmonary consolidation. However, there were some patients who could not have bronchoscopy with suspected VAP because of a variety of safety issues that precluded bronchoscopy. Another limitation is that a substantial proportion of patients (73\%) were treated with antibiotics at the time of the BAL, which may have caused false negative microbiology and, perhaps, even falsely reduced biomarker levels. However, in clinical practice, the reality is that the majority of patients in whom VAP is considered will already be receiving antibiotics. Another issue concerns the cut-off for defining VAP, namely $>10^{4}$ colony-forming units in the BAL fluid. The correct gold standard for diagnosis of VAP is not established, but this was a reasonable approach.

In summary, the study by Hellyer et al provides new data regarding the potential use of low levels of BAL concentrations of IL- $1 \beta$ and IL- 8 to exclude the diagnosis of VAP in critically ill, ventilated patients. Reliable point-of-care measurements are now needed to test prospectively these two biomarkers in a multicentre study to determine the effect on reducing the unnecessary use of antibiotics in suspected VAP. A study of this kind could also assess the relative economic impact of more fiberoptic bronchoscopies versus reduced use of antibiotics in patients in whom VAP could be ruled out, pending the results of microbiological cultures. This approach might have particular value also in ventilated patients who already have pre-existing pulmonary radiographic abnormalities from ARDS, pulmonary trauma and post-operative atelectasis. 


\section{Editorial}

Acknowledgements MAM was supported in part by NHLBI R37 HL51856. Also, my appreciation to Andrew Manies for his assistance in preparing the manuscript.

Funding US Department of Health and Human Services-National Institutes of Health R37 HL51856.

\section{Competing interests None.}

Provenance and peer review Not commissioned; internally peer reviewed.

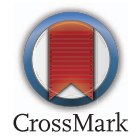

To cite Matthay MA. Thorax 2015;70:5-6.

Published Online First 28 October 2014

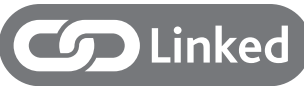

- http://dx.doi.org/10.1136/thoraxjnl-2014-205766
Thorax 2015;70:5-6.

doi:10.1136/thoraxjnl-2014-206280

\section{REFERENCES}

1 Melsen WG, Rovers MM, Groenwold RH, et al. Attributable mortality of ventilator-associated pneumonia: a meta-analysis of individual patient data from randomised prevention studies. Lancet Infect Dis 2013:13:665-71.

2 Klompas M. Interobserver variability in ventilatorassociated pneumonia surveillance. Am J Infect Control 2010;38:237-9.

3 Skrupky LP, McConnell K, Dallas J, et al. A comparison of ventilator-associated pneumonia rates as identified according to the National Healthcare Safety Network and American College of Chest Physicians criteria. Crit Care Med 2012;40:281-4.

4 Kollef MH. Diagnosis of ventilator-associated pneumonia. N Engl J Med 2006;355:2691-3.

5 Klein Klouwenberg PM, van Mourik MS, Ong DS, et al. Electronic implementation of a novel surveillance paradigm for ventilator-associated events. Feasibility and validation. Am J Respir Crit Care Med 2014;189:947-55.

6 Wunderink RG. Ventilator-associated complications, ventilator-associated pneumonia, and Newton's third law of mechanics. Am J Respir Crit Care Med 2014;189:882-3.

7 Chastre J, Fagon JY. Ventilator-associated pneumonia. Am J Respir Crit Care Med 2002;165:867-903.

8 Hellyer T, McAuley DF, Walsh T, et al. Diagnostic accuracy of pulmonary host inflammatory mediators in the exclusion of ventilator-acquired pneumonia. Thorax 2015:70:41-7.

9 Song Y, Lynch SV, Flanagan J, et al. Increased plasminogen activator inhibitor-1 concentrations in bronchoalveolar lavage fluids are associated with increased mortality in a cohort of patients with Pseudomonas aeruginosa. Anesthesiology 2007;106:252-61.

10 Fagon JY. Biological markers and diagnosis of ventilator-associated pneumonia. Crit Care 2011;15:130. 